\title{
Accuracy Analysis on Improved Design of the Plug Back Cover based on Reverse Engineering
}

\author{
Jun Fan ${ }^{1, a}$, Leigang Wang ${ }^{2}$ \\ ${ }^{1}$ Suqian College, $\mathrm{CHN}$ \\ 2 Jiangsu University, $\mathrm{CHN}$
}

\begin{abstract}
Improved design process consists of four steps. These steps are prototype step, design step, analysis step and mold step, respectively. The error constitution of these steps is analyzed. Through the error analysis on both improved design process and reverse process, the total error of reverse improved design is essentially formed by reverse error, design error, analysis error and mold error. Focus on the error structure of reverse improved design of the plug back cover, the accuracy study and control on the whole process is implemented.
\end{abstract}

\section{Introduction}

With the improvement of product requirements, research and development of many products are based on local modification design[1-6]. Under the help of reverse engineering technology, product research and development level enhances unceasingly[7-9], but the accuracy analysis and control is still one of the main factors of restricting the product upgrade[10-11]. In this paper, error constitution of improved design process and error structure of reverse improved design process are studied. Meanwhile, a new approach of precision assessment on reverse improved design is provided. Accuracy of the reverse improved design process is controlled effectively.

\section{Study on Improved Design Process}

\subsection{Step Analyzing}

Improved design process consists of four steps. These steps are prototype step, design step, analysis step and mold step, respectively. The steps of improved design process is shown as Figure 1. The prototype step contains drawing section and representation section. The prototype step is shown as Figure 2. The design step contains modification section and optimization section. The design step is shown as Figure 3.The analysis step contains performance section and mechanism section. The analysis step is shown as Figure 4.The mold step contains structure section and process section. The mold step is shown as Figure 5.

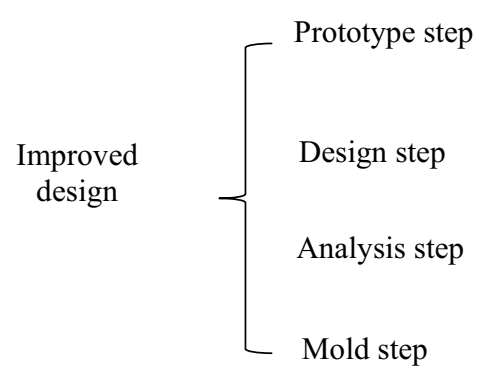

Figure 1. Improved design.

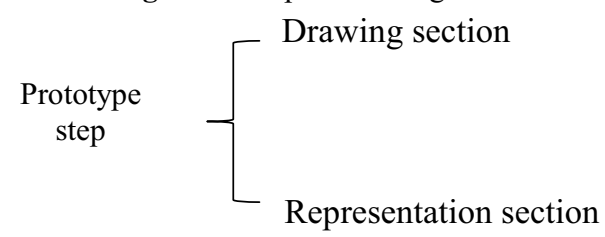

Figure 2. Prototype step.

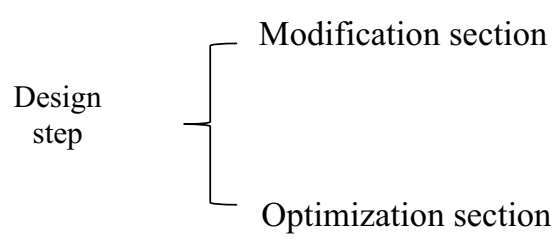

Figure 3. Design step.

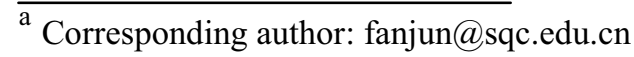




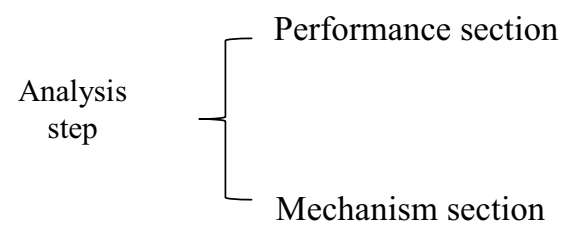

Figure 4. Analysis step.

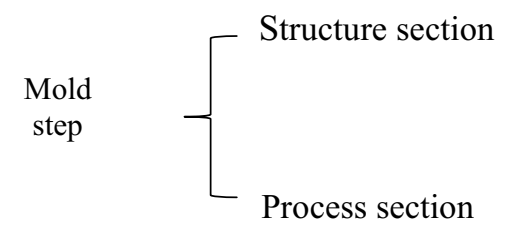

Figure 5. Mold step.

\subsection{Error Constitution}

In the improved design process, the total error is constituted chiefly by prototype error, design error, analysis error and mold error. The error of improved design is shown as Figure 6. The prototype error relates drawing error and representation error. The prototype error is shown as Figure 7. The design error relates modification error and optimization error. The design error is shown as Figure 8. The analysis error relates performance error and mechanism error. The analysis error is shown as Figure 9. The mold error relates structure error and process error. The mold error is shown as Figure 10.

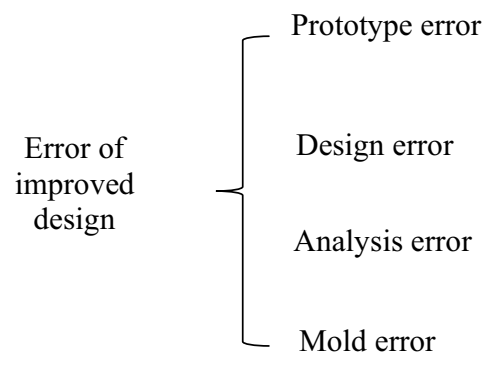

Figure 6. Error of improved design.

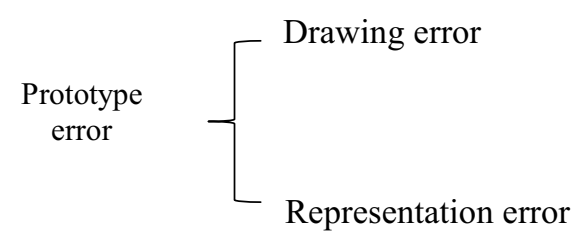

Figure 7. Prototype error.

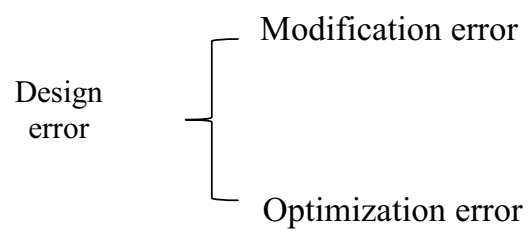

Figure 8. Design error.

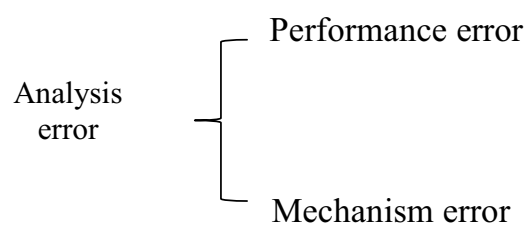

Figure 9. Analysis error.

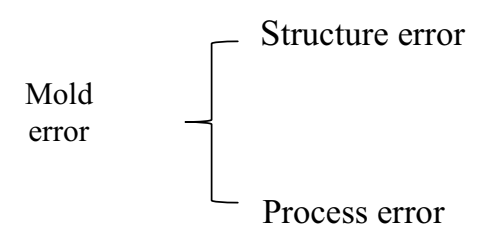

Figure 10. Mold error.

\section{Precision Research on Reverse Improved Design}

\subsection{Error Analysis on Reverse Process}

Reverse process is segmented into three stages, which incorporate acquisition stage, adjustment stage and reconstruction stage. Point cloud is very important in both the acquisition stage and the adjustment stage. Quality of the reverse process is also controlled by these stages. The stages of reverse process is shown as Figure 11 .

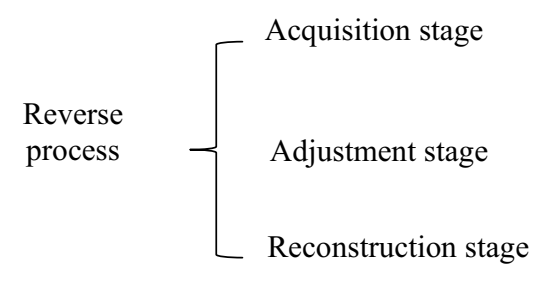

Figure 11. Reverse process.

Based on the stage analysis on reverse process, error of reverse process is composed of the acquisition error, the adjustment error and the reconstruction error. The reverse error is shown as Figure 12. 


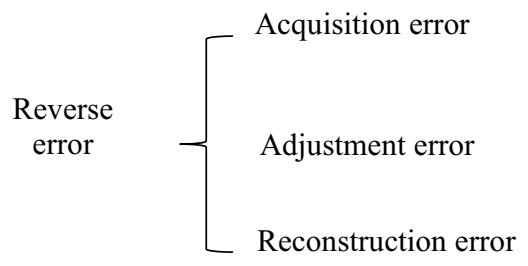

Figure 12. Reverse error.

\subsection{Error Structure of Reverse Improved Design}

The process of reverse improved design includes reverse step, reverse design step, reverse analysis step and reverse mold step. The steps of reverse improved design is shown as Figure 13. The reverse design step contains reverse modification section and reverse optimization section. The reverse design step is shown as Figure 14. The reverse analysis step contains reverse performance section and reverse mechanism section. The reverse analysis step is shown as Figure 15. The reverse mold step contains reverse structure section and reverse process section. The reverse mold step is shown as Figure 16.

Through the error analysis on both improved design process and reverse process, the total error of reverse improved design is essentially formed by reverse error, reverse design error, reverse analysis error and reverse mold error. The error of reverse improved design is shown as Figure 17. The reverse error comprises acquisition error, adjustment error and reconstruction error. The reverse design error comprises reverse modification error and reverse optimization error. The reverse design error is shown as Figure 18. The reverse analysis error comprises reverse performance error and reverse mechanism error. The reverse analysis error is shown as Figure 19. The reverse mold error comprises reverse structure error and reverse process error. The reverse mold error is shown as Figure 20.

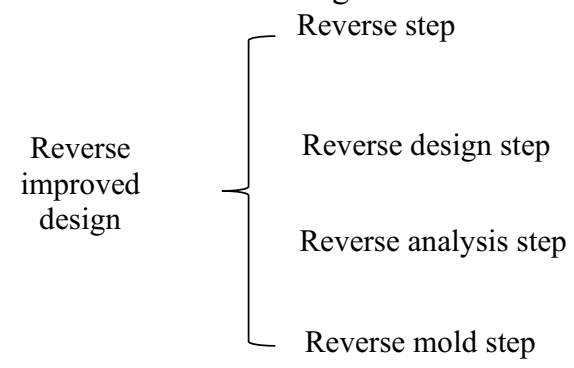

Figure 13. Reverse improved design.

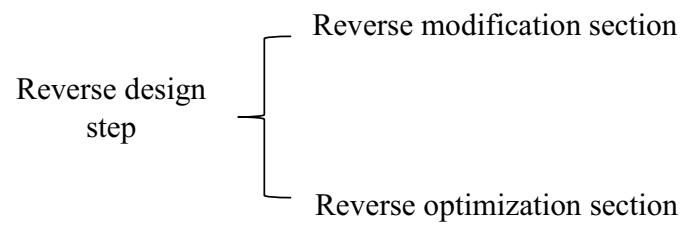

Figure 14. Reverse design step.

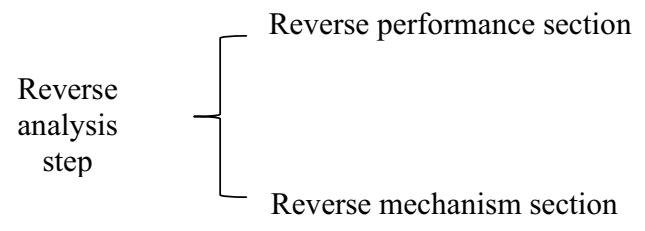

Figure 15. Reverse analysis step.

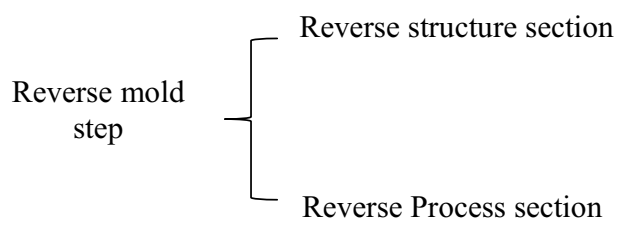

Figure 16. Reverse mold step.

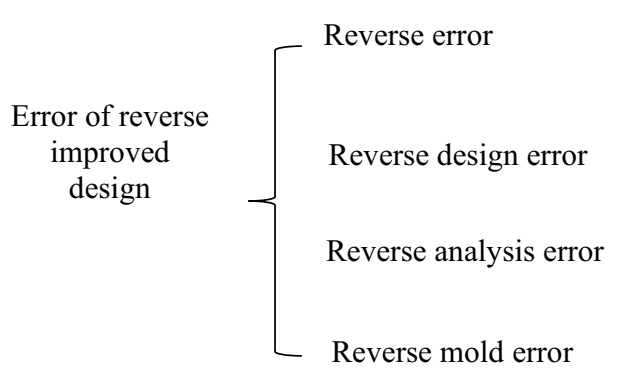

Figure 17. Error of reverse improved design.

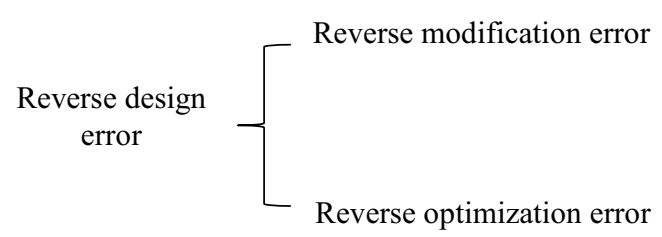

Figure 18. Reverse design error.

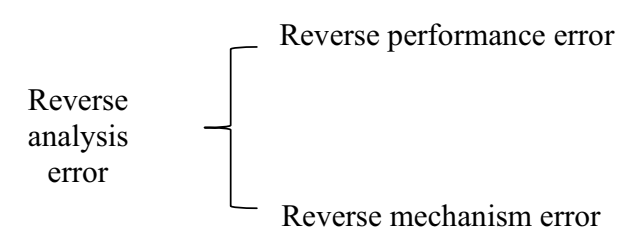

Figure 19. Reverse analysis error.

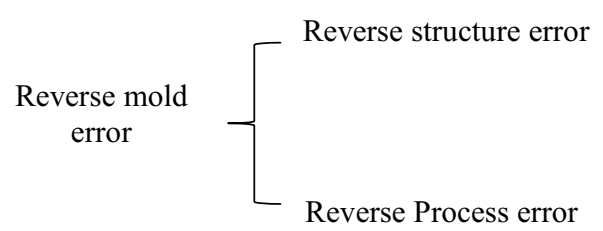

Figure 20. Reverse mold error. 
The total error formula of the process is presented in Equation 1.

$$
\Delta_{\text {tatal }}^{2}=\Delta \underset{\Delta_{\text {reverse }}^{2}}{2}+\Delta_{\text {reverse mold }}^{2} \text { reverse design }+\Delta_{\text {reverse analysis }}^{2}+
$$

In Equation 1, the reverse error formula is presented in Equation 2.

$\Delta_{\text {reverse }}^{2}=\Delta_{\text {acquisition }}^{2}+\Delta_{\text {adjustment }}^{2}+\Delta_{\text {reconstruction }}^{2}$

In Equation 1, the reverse design error formula is presented in Equation 3.

$\Delta^{2}{ }_{\text {reverse design }}=\Delta{ }^{2}$ reverse modification $+\Delta^{2}$ reverse optimization

In Equation 1, the reverse analysis error formula is presented in Equation 4.

$\Delta{ }_{\text {reverse analysis }}^{2}=\Delta{ }_{\text {reverse performance }}^{2}+\Delta{ }_{\text {reverse mechanism }}^{2}$

In Equation 1, the reverse mold error formula is presented in Equation 5.

$\Delta{ }_{\text {reverse mold }}^{2}=\Delta_{\text {reverse structure }}^{2}+\Delta_{\text {reverse process }}^{2}$

\section{Case}

Figure 21 shows point set of a plug back cover,which needs to be reverse improved designed. The process of the reverse improved design error is required lower than $0.1 \mathrm{~mm}$.

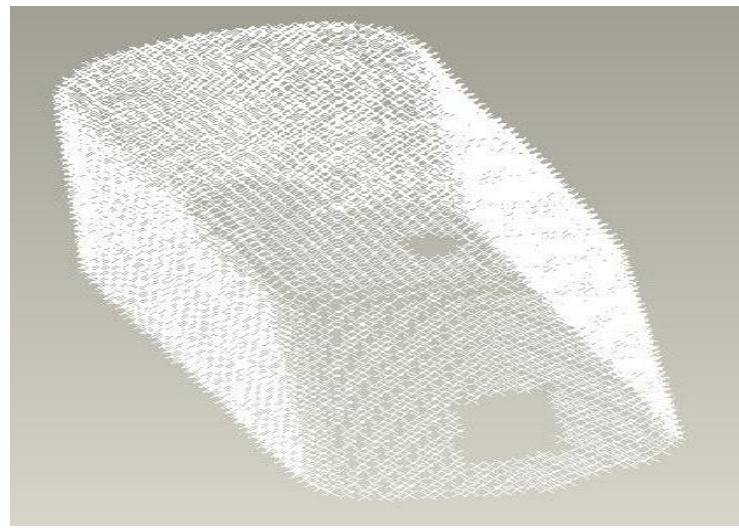

Figure 21. Point set.

The reconstruction product is shown in Figure 22. The reverse error is confined to about $0.059 \mathrm{~mm}$. The reverse modification error and the reverse optimization error are shown in Figure 23 and Figure 24, respectively. In the light of Equation 3, the reverse design error is limited to about $0.0001 \mathrm{~mm}$. Reverse performance analysis is shown in Figure 25.

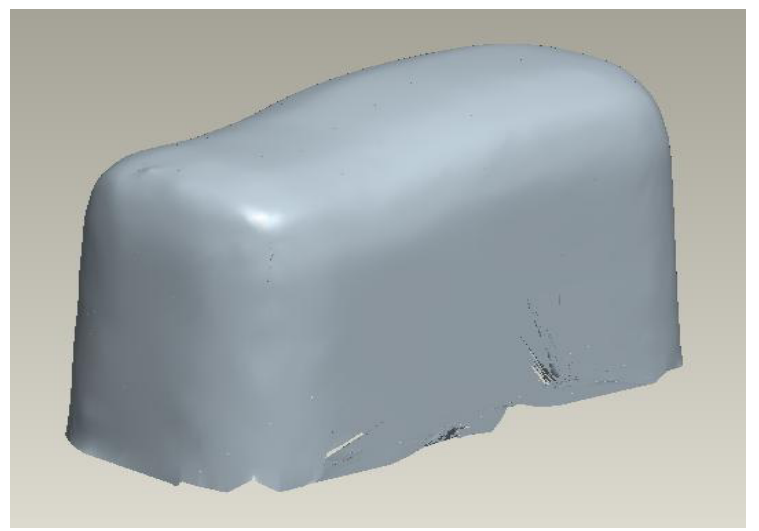

Figure 22. Reconstruction.
The reverse analysis error and the reverse mold design error, is controlled at about $0.014 \mathrm{~mm}$ and $0.022 \mathrm{~mm}$, respectively. Under Equation 1, the total error of the reverse improved design is $0.065 \mathrm{~mm}$, which is not exceed the standard of $0.10 \mathrm{~mm}$. Hence, the accuracy of the reverse improved design has been controlled.

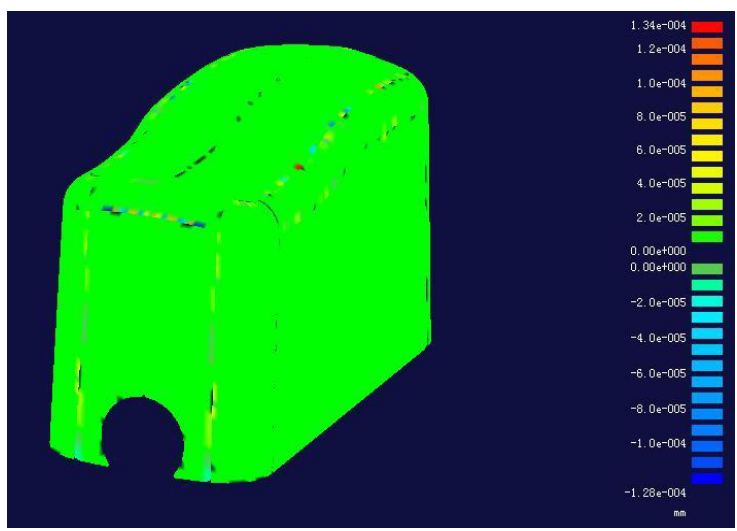

Figure 23. Reverse modification error.

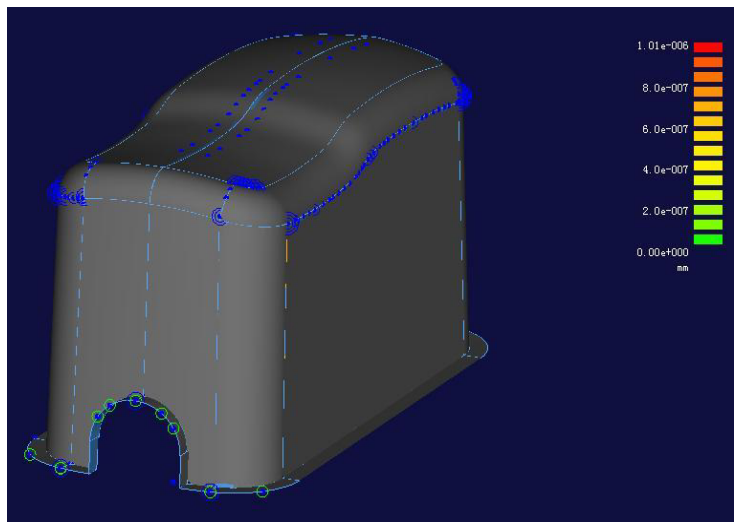

Figure 24. Reverse optimization error .

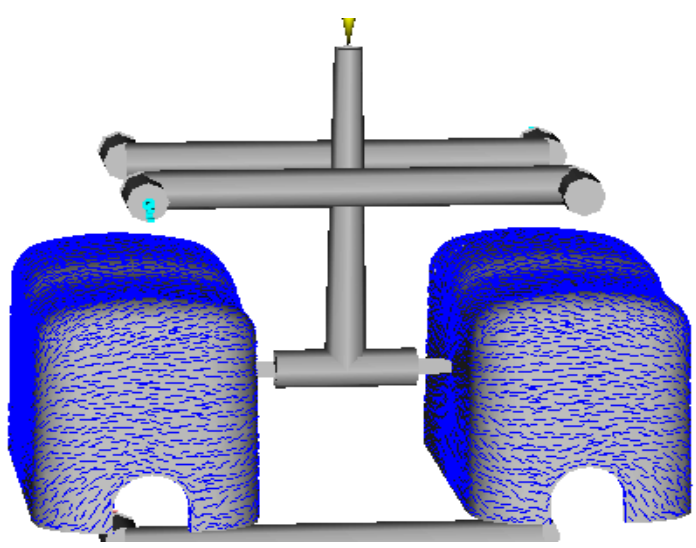

Figure 25. Reverse performance analysis.

\section{Conclusions}

Accuracy control on reverse improved design of the plug back cover based on error analysis is realized. For the future industrial application, this approach will provide a practice basis for reverse development design of products. 


\section{Acknowledgements}

This study was provided financial aid by the Scientific Research Project of Suqian College(2015KY30), the Jiangsu Brand Professional Construction Engineering Project in Colleges and Universities(PPZY2015C252), the Student Innovation Training Project of Suqian College in 2015.

\section{References}

1. Xiaobo Shi. Manufacturing Automation, 32,122. (2010)

2. Qiang Huang. Coal Mine Machinery. 32,12. (2011)

3. Jun Fan, Leigang Wang. Applied Mechanics and Materials.494-495,328(2014)

4. Xuehui Chen, Lei Huang, Jingfa Lei, et al. Coal Mine Machinery.32,57. (2011)

5. Yuan Wang, Jianguang Fang, Jingren Wang, et al. Automotive Engineering. 34,847. (2012)

6. Shuhong Ge, Ding Fan, Kai Peng. Computer Simulation. 29,81.(2012)

7. Jun Fan, Leigang Wang. Applied Mechanics and Materials.385-386,121(2013)

8. Jun Fan, Leigang Wang. Applied Mechanics and Materials.538,83( 2014)

9. Jun Fan, Leigang Wang. Manufacturing Automation. 35,131.(2013)

10. Jun Fan, Leigang Wang. Machine Tool \& Hydraulics. 41, 74.(2013)

11. Jun Fan, Leigang Wang. Manufacturing Automation. 35,26.(2013) 